\title{
IR optical system design of uncooled thermal imaging camera in long band $(8-12 \mu \mathrm{m})$
}

\author{
Aboobida Y.A. Haj noor ${ }^{1}$, Faiz M. B. Elshafia ${ }^{2}$, Mubarak M. Ahmed ${ }^{2,3}$ \\ ${ }^{I}$ ZEC Company Cooperation \\ ${ }^{2}$ Institute of Laser, Sudan University of Science and Technology, Khartoum, SUDAN \\ ${ }^{3}$ Department of Physics, College of Science and Arts Al-Muthnib, Qassim University, SAUDI ARABIA
}

\begin{abstract}
For $384 \times 288$ pixel uncooled micro bolometer (a-Si) focal plane array (FPA) detector an infrared optical system (lenses) has been designed in this paper for work in long wavelength infrared region (LWIR) 8 $12 \mu \mathrm{m}$. the design results show that the optimum optical performance can be obtained by our proposed approach, so the modulation transfer function (MTF) at Nyquist frequency above 0.5 and closed to diffraction limit, and diameter of geometrical blur image less than detector unit cell (pixel) and concentrated on it. The main advantages of this design beside quality of it optically, it's simple in structure (consists of one conic surface - ellipse; and five spherical surfaces), lightweight (about 77.59g), small volume (entrance pupil diameter $=40 \mathrm{~mm}$ ), and short length (total track $=58.5 \mathrm{~mm}$ ). These mechanical parameters in addition to chosen tolerance level for manufacture of lenses have a positive affect on the cost of production for this designed system. So the designed optical system should good enough to meets the technical performance requirements for: high image quality, small size, light weight, and low cost thermal camera. This system can be applied in many civilian applications, and short range military applications.
\end{abstract}

Keywords: thermal imaging camera; Zemax; Modulation Transfer Function (MTF); Nyquist frequency; spot diagram; total track; diffraction limit; aspheric surface.

\section{Introduction:}

An infrared camera or thermal imaging camera is a device that forms an image using infrared radiation emitted by object and its surrounding. Due to its reliance on variation of self-emitted radiation of scene contents rather than reflected radiation. It is superior to conventional cameras in many respects. It can work at any level of visibility, i.e. operate both in day and night conditions and absolute darkness and in poor or bad weather conditions, i.e., they can penetrate smoke, dust, fog and light rain ${ }^{[1]}$. The optical system in thermal imaging system used for collecting the maximum amount of radiated energy from a scene containing the target and project image of the scene onto the system detector array. IR optical system is most important subsystem of thermal camera, because it plays a key role in defining some of the overall system parameters like system resolution, system field of view ${ }^{[2]}$. IR optical systems offer long ranges and better see through capability, for this reason its preferred choice over image intensifier tube for civil services and military forces ${ }^{\left[{ }^{[3]}\right.}$.

On the one hand, designing of IR optical systems easier than visible optical systems, since index of refraction of most IR materials is higher, the wavelengths of IR are longer, and their relative dispersion is lower, this generally result in reduction of primary aberrations ${ }^{[4]}$, but on the other hand designing of IR optical system featuring by other critical considerations not considered in other design type of optical imaging system such as: pixel size V/s diffraction spot, IR materials of lenses is rare and limited and expensive, vignetting, cold shield efficiency, and thermalization etc, these additional factors make the design of IR optical system more complex $[3,4]$.

There are four basic goals in design of an optical systems, the $1^{\text {st }}$ goal is to maximize overall system performance, the $2^{\text {nd }}$ goal is to maximize the resolving power for the desired task while maintaining good area coverage, the $3^{\text {rd }}$ goal is to maximize the amount of image flux that is collected and $4^{\text {th }}$ goal is to minimize system complexity, cost, weight ${ }^{[2]}$.

In this work we focus on $4^{\text {th }}$ goal and taking in account all other goals that confined in quality of the final image obtained by it to reduce the cost, weight and assembly complexity of the optical system in order to make it suitable for the number of civilian applications

Therefore the main purpose of this paper is design of infrared optical system to operate in the 8-12 $\mu \mathrm{m}$ wavelength band; it features by low cost and high image quality for thermal imaging purpose.

\section{2-1 Design criterions}

\section{Design and Experimental Work:}

In this work, for design a good optical system from optical, mechanical and financial aspects, there are some criterions (limitations) must be met. Optically, the design limits used is that the diffraction blur (spot 
diagram) should be less than single pixel of detector (less than $25 \mu \mathrm{m}$ ) as much as possible, and the polychromatic diffraction Modulation Transfer Function (MTF) at Nyquist frequency should be above 0.5 and closed to diffraction limit (free aberration) ${ }^{[5]}$. Other criterions required in the designed IR optical system is that: compact design (light weight, small size, and short length), simple in structure, and finally low-cost.

\section{2-2 Basic design parameters (technical parameters)}

This design of IR optical system is based on GUIDIR Company's production of $384 \times 288$ pixels uncooled micro bolometer (a-Si) focal plan array detector module (IR113), the pixel size is $25 \mu \mathrm{m} \times 25 \mu \mathrm{m}$, and the material of detector window (filter) is germanium. Important parameters of the thermal detector represents the optical interface of it which are to be considered in design of optical system are number of pixels, pixel size, thickness of detector window (filter), material of it, and distance between detector window and FPA. If any one of these parameters is changed this leads to failure of optical system ${ }^{[3]}$. The basic parameters of optical system are shown in table (1).

Table (1): basic parameters of optical system

\begin{tabular}{|c|c|}
\hline Wave band (spectral range) & $8 \sim 12 \mu \mathrm{m}$ \\
\hline Effective focal length (EFFL) & $45 \mathrm{~mm}$ \\
\hline F/number & 1.125 \\
\hline FOV(field of view = HFOV $\times$ VFOV) ${ }^{1}$ & $12.18^{\circ} \times 9.15^{\circ}$ \\
\hline Entrance pupil diameter $^{2}$ & $40 \mathrm{~mm}$ \\
\hline Total track $^{2}$ & $58.5 \mathrm{~mm}$ \\
\hline Detector window thickness & $1 \mathrm{~mm}$ \\
\hline Distance from window to FPA & $3.15 \mathrm{~mm}$ \\
\hline
\end{tabular}

\section{2-3 Design procedure}

The previous basic design parameters meet the target requirements of optical design if at least the design criterions (limits of design) that identified previously are achieved. In the design and optimization phases of the optical system there are a number of tasks that the designer must perform and consider [6].

\footnotetext{
${ }^{1}$ This parameter (FOV) is depending on EFFL of the system and dimension of the detector in vertical and horizontal directions.

- $\mathrm{EFFL}=45$.

- Horizontal detector dimension $=$ Horizontal number of pixel $\times$ pixel pitch $=384 \times 0.025=9.6$.

- Vertical detector dimension $=$ Vertical number of pixels $\times$ pixel pitch $=288 \times 0.025=7.2 \mathrm{~mm}$.

- $\mathrm{HFOV}=2 \tan ^{-1}\left(9.6 / 2 \times 45^{2}\right)=12.18^{\circ}$.

$-\mathrm{VFOV}=2 \tan ^{-1}\left(\mathrm{C}^{7.2} / 2 \times 45^{2}\right)=9.15^{\circ}$.
}

${ }^{2}$ The length of the optical system (TOTR) is depending on EEFL also; there is no precise relation between these parameters (TOTR\& EFFL), for this work because we need compact optical system as mechanical consideration. For this reason and from my previous work in this field we chose the TOTR $=1.3 \times \mathrm{EFFL}=$ $1.3 \times 45=58.5 \mathrm{~mm}$.

Figure (1) shows just outlines of the basic steps followed by designer in the design task of optical system.

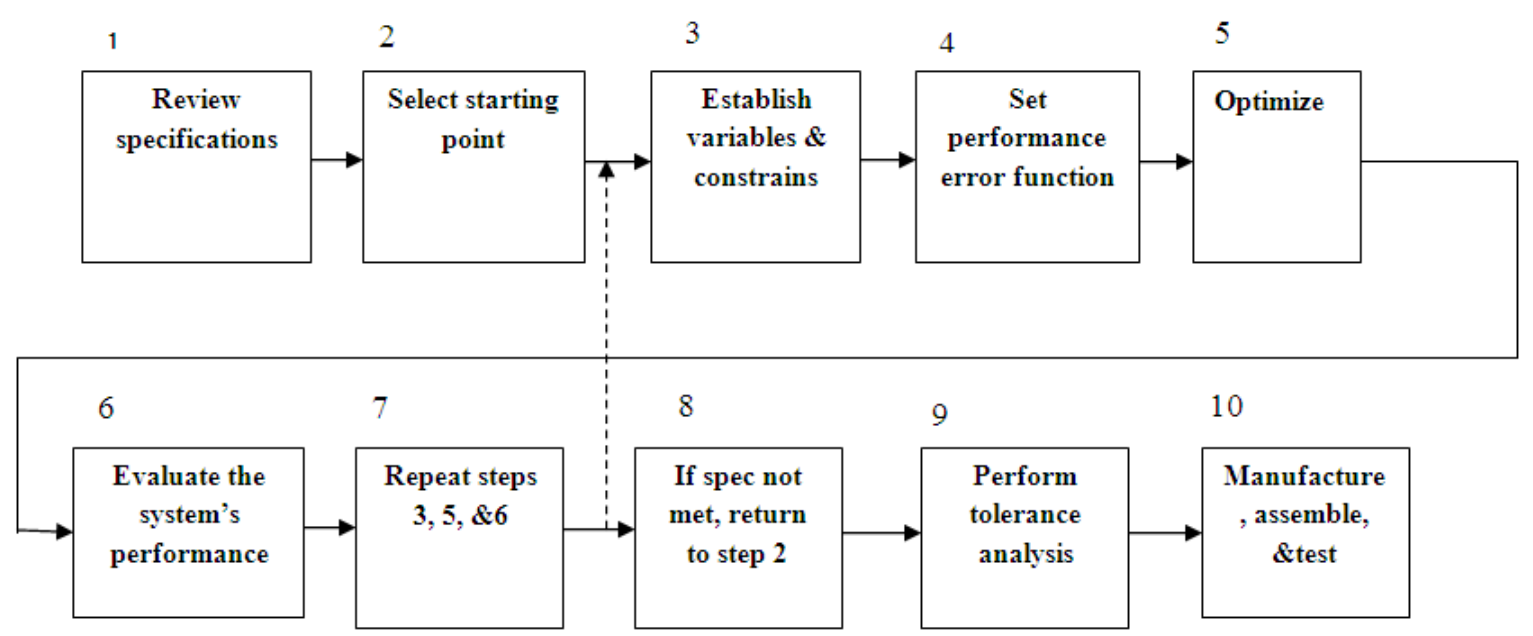

Fig (1): optical system design procedure ${ }^{[6]}$ 
To design, optimize and analyze the IR optical system for thermal camera we choose ZEMAX software program because it's a comprehensive software tool. It integrates all the features required to conceptualize, design, optimize, analyze, tolerance, and document virtually any optical system. It is widely used in optics industry as standard design tool ${ }^{[7]}$.

To get the final design there are many critical and necessary steps in software package (ZEMAX) should be done carefully, the sequence of these steps briefly are:

- Entered the starting point into the software package (ZEMAX) and setting the basic parameter of the system like system aperture, lens unit, FOV, operating wavelength, and entered the information about lenses itself like (surface type, surfaces radius of curvatures, elements thickness, material, etc).

- After that optimized the start point to achieve the final design, this step (optimization) comes after determined the degree of freedom and select appropriate variables for optimization, and created of default merit function (operands within merit function define the goal which you are attempting to achieve by optimization).

- Evaluated the system performance by analysis features available in ZEMAX and ensure that all the initial design constraints are met ${ }^{[8]}$.

- Repeated the last two steps (optimization, and evaluation) frequently by true understanding and made many adjustments until the desired performance is met, thus, the final design was constructed.

By following the previous steps through ZEMAX optical design program the IR optical system was designed after many optimization cycles. The specifications of constructed design based on IR113 thermal detector module are shown in table (2)

Table (2): final design specifications

\begin{tabular}{|l|l|}
\hline & \multicolumn{1}{|c|}{ Detector } \\
\hline Detector material & UFPA micro bolometer, Asi \\
\hline Spectral range & $8 \sim 12 \mu \mathrm{m}$ \\
\hline Resolution (pixels) & $384 \times 288$ \\
\hline Noise Equivalent Temp Difference NETD & $<100 \mathrm{mK} @ 30^{\circ} \mathrm{C}$ \\
\hline Pitch & $25 \mu \mathrm{m} \times 25 \mu \mathrm{m}$ \\
\hline Thermal response time & $7 \mathrm{~ms}$ \\
\hline Fill factor & $>80 \%$ \\
\hline Bad pixel & $<1 \%$ \\
\hline & Optics \\
\hline Lens & $45 \mathrm{~mm}$ \\
\hline F\# & 1.125 \\
\hline FOV & $12.18^{\circ} \times 9.15^{\circ}$ \\
\hline Focus & $5 \mathrm{~m}$ to infinity \\
\hline
\end{tabular}

Figures (1-a), (1-b), and (1-c) show 2D, 3D, and shaded model layout diagrams of this designed optical system

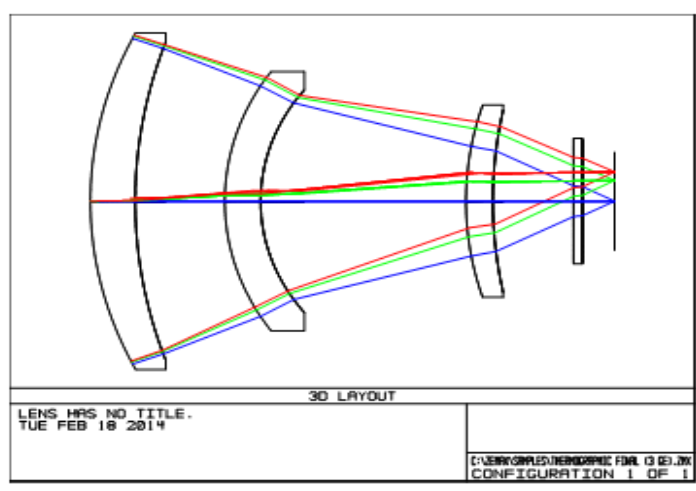

Fig (1-a): 2D layout

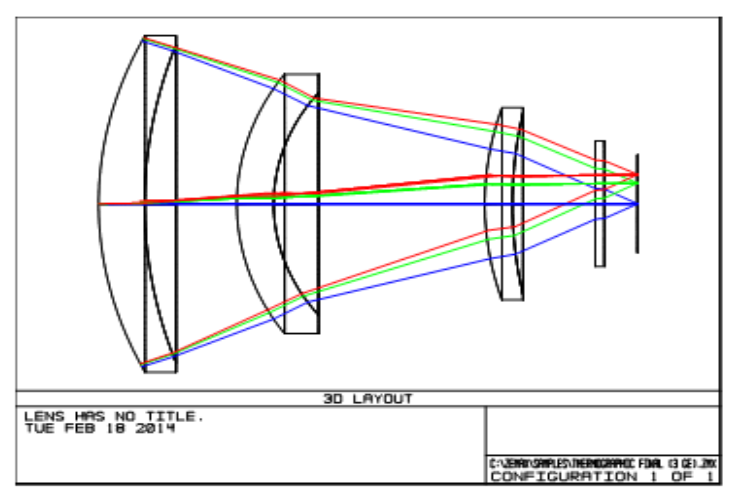

Fig (1-b): 3D layout 


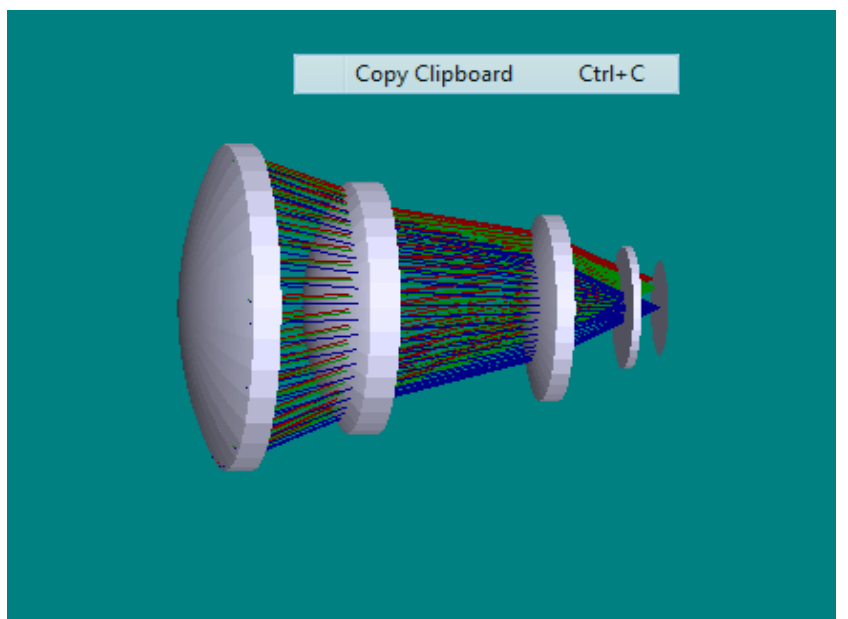

Fig (1-c): shaded model layout

\section{Results and Discussion:}

* The designed optical system consists of 3 lenses made from germanium (monocrystal n-type) material the advantages' of this material

- Has high refractive index (>4.003) and high optical power, this leads to a smooth lens curvature for imaging.

- Has high mechanical strength (good hardness and hence scratch resistance) and made easily in any shape and size by diamond turning machine. It is therefore considered to be the best material for infrared use.

- Has much better refractive index homogeneity $(0.1-1.0) \times 10^{-4}$.

- Has low spherical aberration, low chromatic aberration ${ }^{[1]}$. As shown in fig (6); often no correction need.

The drawback of germanium, the change in refractive index with respect to temperature relatively high $(\mathrm{dn} / \mathrm{dt} \text { is high })^{[1]}$, this is not critical problem in this design for two reasons:

i. This system is designed for civilian applications, which mean it works at normal level of temperature where the variations in temp not high hence not affect in quality of final image.

ii. The defocus problem come from variation in distance between camera and moving object, and variation of lenses refractive index with temperature $(\mathrm{dn} / \mathrm{dT})$ was resolved well mechanically by moving the $3^{\text {rd }}$ lens axially and uses it as focus lens in order to compensate for image location shift (defocus) caused by movement of object and temperature change ${ }^{[9]}$. we found the minimum distance of object form camera to achieve clear image about $5 \mathrm{~m}$, this happens by moving $3^{\text {rd }}$ lens (focus lens) about $0.5 \mathrm{~mm}$ towards $2^{\text {nd }}$ lens. The movement of focus lens $(0.5 \mathrm{~mm})$ according to the object location was calculated accurately from accounts tables.

* All surfaces of lenses in designed optical system are spherical surfaces with exception of the first surface, it is simple aspheric (ellipse), and the use of aspheric surfaces in optical design provides added design variables without increasing the number of optical components and leads to make the design ${ }^{[1]}$ :

- Better in aberration correction

- Better in transmission

- $\quad$ Light in weight

- Cheap raw materials

- And more compact design

* The field of view of designed system is large or wide $\left(\mathrm{FOV}=12.18^{\circ} \times 9.15^{\circ}\right)$, this allows the sensor to view or cover large area.

* F/number of system is low $(\mathrm{F} \#=1.125)$, therefore the speed of designed system is high. Thus his ability to capture images for quick targets.

* There are many analysis (diagnostic) tools, either graphic or text in ZEMAX program which can be used to analyze and measure optical performance of obtained optical system. In this work we used some of these analysis tools (more important and useful from my point of view) to evaluate the final design of optical system, like:

1. Spot diagram: it's very useful diagnostic (analysis) tool in ZEMX. The spot diagram is the geometrical image blur formed by the lens when imaging a point object ${ }^{[6]}$, by default ZEMAX plots diagram for each field point. In this design one of the most important criterion (limit) used in creation of the design is that the diffraction blur (spot diagram) of point source should be less than single pixel of detector (less than $25 \mu \mathrm{m}$ ) and concentrate on it as much as possible, because the electro-optic system resolution depends on the optical 
blur diameter and size of single detector element (pixel). As shown in fig (3) the black box surrounding the spot represents the dimension of the pixel (box width $=25 \mu \mathrm{m}$ ), for all field points (on-axis, $0.7 \mathrm{FOV}$, and full FOV) the target criterion is met in this design.

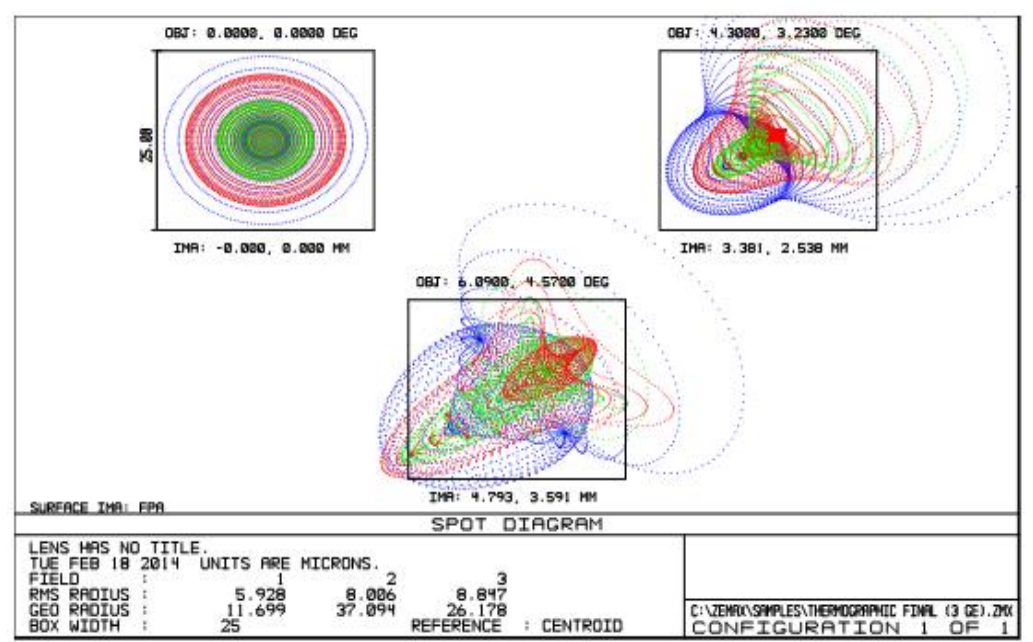

Fig (3): spot diagram on $25 \mu \mathrm{m}$ pixel

2. Modulation Transfer Function (MTF): it is best and more comprehensive way to evaluate an optical system, especially for imaging (image forming) systems, this analysis tool describes the ability of the optical system to distinguish the details; it used as measure to the resolution (To what extent can distinguish between details) of optical system; In general the wider the MTF the better the resolution ${ }^{[10]}$.

Polychromatic MTF used as criterion (limit) in this design, the target value of MTF for all field points above 0.5 and MTF for on-axis and 0.7FOV near to diffraction limit. As shown in fig (4) the lower value of MTF at Nyquist frequency (detector cut off frequency) 20cycle $/ \mathrm{mm}$ is 0.56 at edge of FOV. And MTF value for on-axis and $0.7 \mathrm{FOV}$ is very near to diffraction limit, so this criterion is met well in the designed optical system.

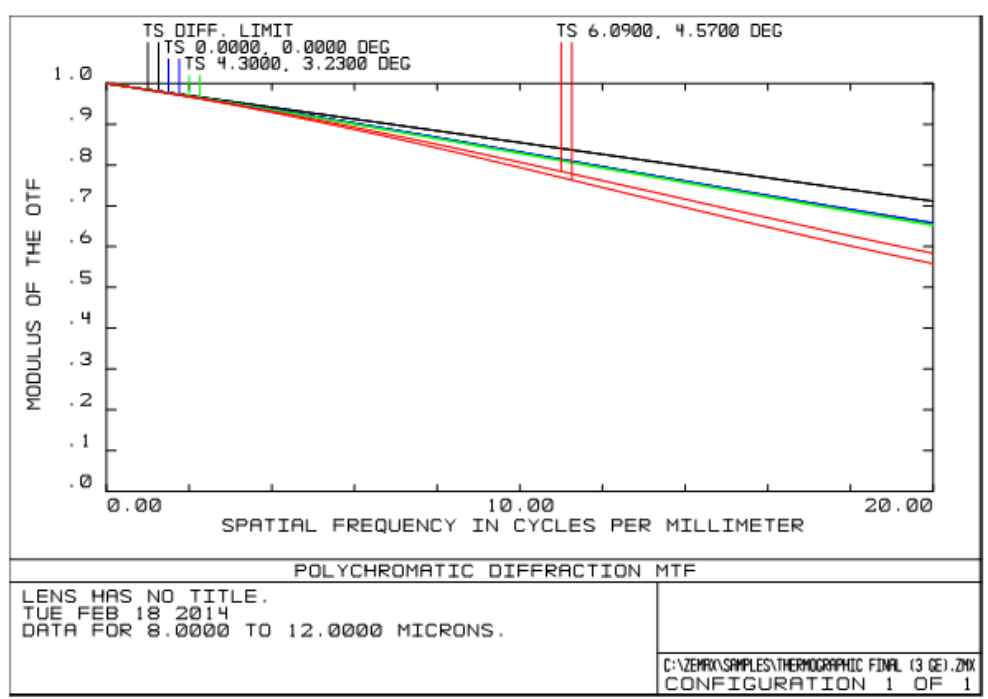

Fig (4): polychromatic diffraction MTF

3. Transverse Ray fan plot (ray aberration): this is one of the most important diagnostic tools in ZEMAX, transverse ray aberration plots is commonly used as tool to assessed the resolution of the optical system. These plots are derived from tracing rays through the optical system and finding out where they arrive at the detector. Transverse aberration plots give an idea of image blur at the detector. The vertical axis of each plot gives the magnitude of the blur. The horizontal axis represents the beam diameter for any field angle and is effectively normalized so dimensions are not given. Thus the transverse aberration plot shows variation in blur size at the detector for a cross-section of rays across an input beam ${ }^{[11]}$. Fig (5) shown that generally the bullring contained within a pixel. 


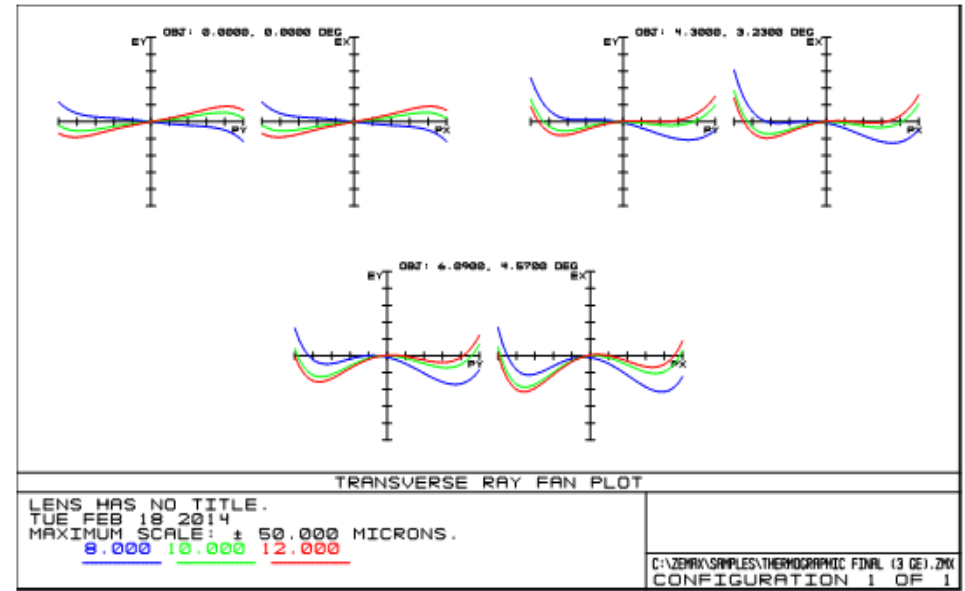

Fig (5): ray fan plot

4. Chromatic focal shift (chromatic aberration): this plot shows the variation in focal length with wavelength because the refractive indexes of optical materials function in wavelength. Fig (6) shown that the maximum focal shift is $27 \mu \mathrm{m}$ appears in minimum wavelength $8 \mu \mathrm{m}$ this is very acceptable value.

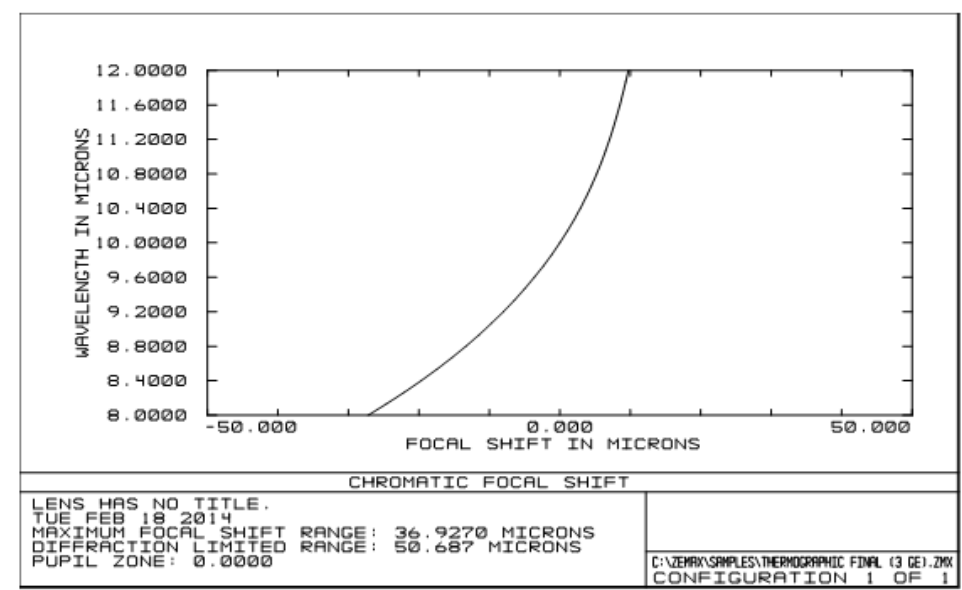

Fig (6): chromatic focal shift

5. Encircled energy: this analysis tool show the energy distribution of blur spot as function of blur spot radius. As shown from fig (7), the lower fraction of enclosed energy is 0.6992 at $12.5 \mu \mathrm{m}$ (half diameter of pixel) occur at edge of FOV. And for anther FOV points on axis\& 0.707FOV the fraction of encircled energy is near to diffraction limit (free aberration-ideal system); this is very acceptable specification in the design.

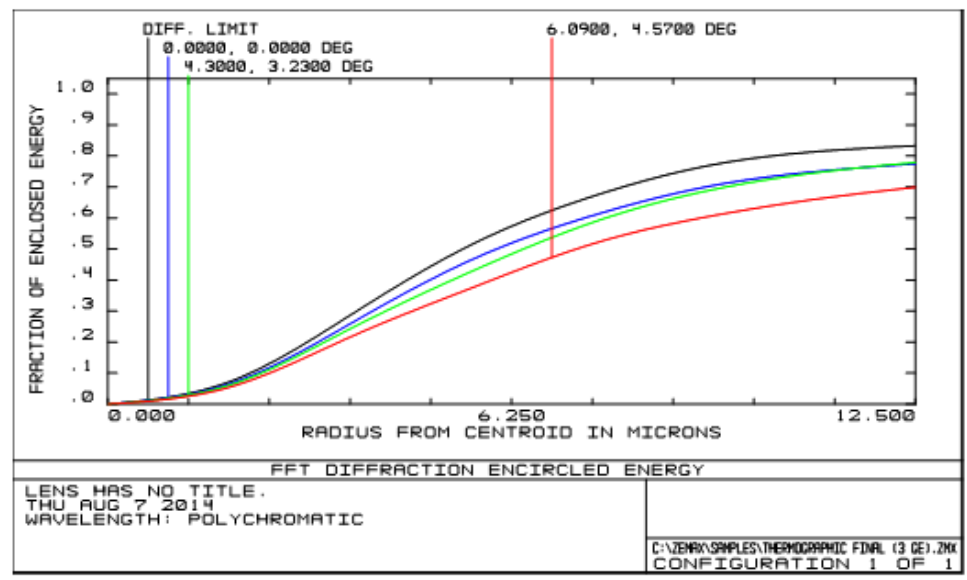

Fig (7): encircled energy 
6. Lateral color: the amount of lateral color aberration in edge of $1 / 2$ maximum diagonal FOV $7.614^{\circ}$ is $1.7683 \mu \mathrm{m}$ as shown in fig (8); this is perfect and acceptable value.

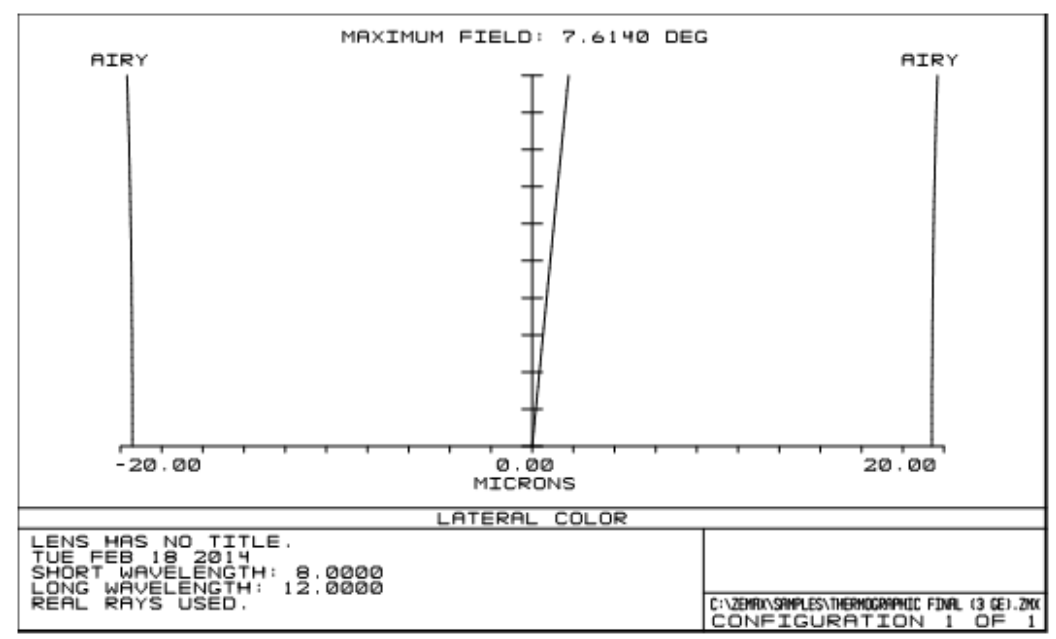

Fig (8): lateral color

According to previous results and analytics the designed optical system met all required specifications from optical point of view (quality of final image). On the other hand, advantages of this design mechanically it is compact design, light weight, and simple in structure. These mechanical features have a positive impact affect on the cost of optical system by reduce the raw material cost and manufacturing cost of the optical system. So this design met all target specifications optically, mechanically, and financially.

The text below show prescription data of designed IR optical system:

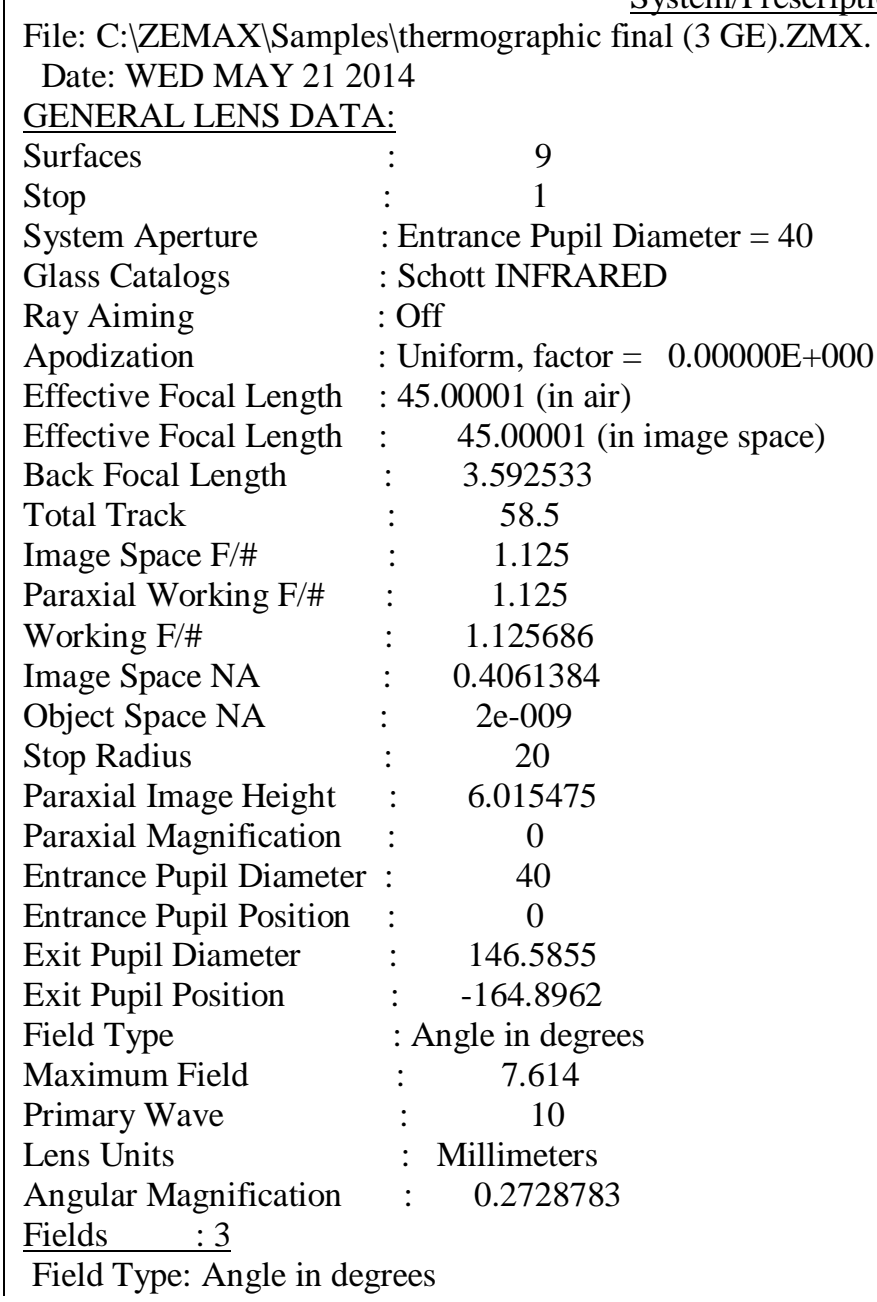


IR optical system design of uncooled thermal imaging camera in long band $(8-12 \mu \mathrm{m})$

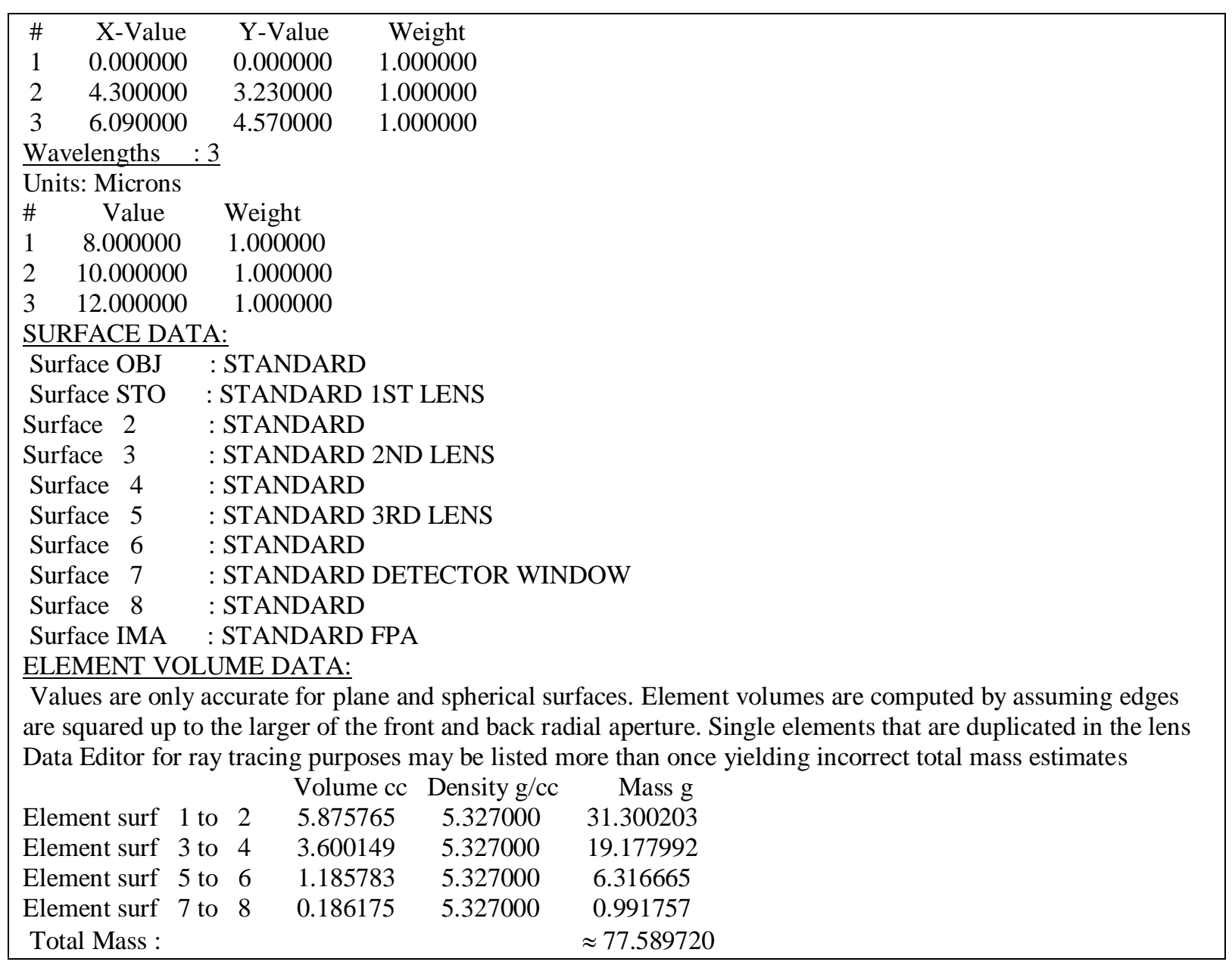

Finally, the tolerance analysis is important step in the design process and must be done before entering into industrialization. Zemax can be very helpful in these situations ${ }^{[12]}$. Tolerances are small errors/imperfections allowed in fabrication and assembly of the optical element, of course there is no optical part can be fabricated or aligned-assembly perfectly ${ }^{[13]}$, by the end of this important and complex analysis we defined error values are allowed in the manufacturing and assembly stages so as not to affect the efficiency of the optical system and sure that by these amount of tolerance values the optical system meet the required level of optical performance at reasonable cost, because the cost of manufacturing well be affected by level of tolerance, and level of tolerance in this design is commercial level that is means we considered the cost during this important process.

\section{Conclusion:}

IR optical system for working compatible with uncooled thermal detector module (IR113) to build thermal imager device was designed. The optical performance of designed optical system is good, so the MTF values for selected field points in Nyquist frequency limit above 0.5 (minimum value of MTF is 0.56 at edge of FOV) and near to diffraction limit curve as shown in fig (4) that is means the design performance is near diffraction limited (free aberration or perfect optical design), and the geometrical image blur formed by the lenses when imaging a point object is concentrated within pixel area, so the maximum RMS radius of image blur (spot diagram) occur at edge of the FOV is $8.847 \mu \mathrm{m}$ as shown in fig (3). These design results shown that the designed optical system has high image quality, and the combination of this optical system with IR detector module will produce image with high quality. The mechanical performance of the designed system is good enough; its light weight, small volume, and simple in structure. These mechanical features have a positive impact on total cost of the optical system (compare with day vision optical system IR optical system is more expensive). This design is suitable for IR imaging system in many applications like thermography for medical applications, nondestructive test, firefighting, search and rescue, night navigation, and short range military\& paramilitary applications.

In future, the mechanical case will be designed, and detector power board will be designed and build, these to transforming to compact hand-held thermal imaging camera. 


\section{References:}

[1]. R N SINGH "THERMAL IMAGING TECHNOLOGY Design and Applications", Universities Press (India) Private Limited [2009].

[2]. Ronald G. Diggers, Paul Cox, Timothy Edwared "Introduction to Infrared and Electro-Optical Systems ", Artech House, INC. Boston London [1999]

[3]. Ikbal Singh "DESIGN OF INFRERED OPTICAL SYSTEM", International Conference on Optics and Photonics, India, [2009].

[4]. Max J. Riedl, "Optical Design Fundamentals for Infrared Systems", Tutorial Texts in Optical Engineering, Volume TT20, ISBN 0-81941935-4. [1995].

[5]. Ramin Khoei "Optical design of a long range dual field of view thermal imaging camera in 3-5 wave band ", Proc. of SPIE Vol. 7506 75061M-1, [2009].

[6]. Robert E. Fischer, Biljana Tadic-Galeb \&Paul R. Yoder "Optical System Design", $2^{\text {nd }}$ Ed, (McGraw-Hill, New York), [2008].

[7]. ZEMAX Optical Design Program, Users Guide, ZEMAX Development Corporation, [9 June 2009].

[8]. http://www.zemax.com/kb/articles/20/1/How-To-Design-a-Singlet-Lens/Page1.html. By Dan Hill - Published on [21 July 2005].

[9]. Allen Mann "INFRARED OPTICS AND ZOOM LENSES" second edition, Tutorial Texts in Optical Engineering, Volume TT83,SPIE PRESS USA, [2009].

[10]. G. M. Koretsky, J. F. Nicoll, \&M. S. Taylor "A Tutorial on Electro-Optical/Infrared (EO/IR) Theory and System" Institute for Defense Analysis IDA Document D-4642, [January 2013].

[11]. "Transverse Ray Aberrations" PRECISION-OPTICAL ENGINERRING, application note OSE 001.

[12]. Rachel Haynes "Tolerancing in Zemax" Opti 521.Tutorial. [December 10, 2007].

[13]. George Curatu, Ph.D. "TOLERANCES IN OPTICAL DESIGN" LensAR, Inc. 2800 Discovery Drive Orlando, FL 32826 [December 6, 2010]. 